\title{
Simultaneous iterative algorithms for the split common fixed-point problem of generalized asymptotically quasi-nonexpansive mappings without prior knowledge of operator norms
}

Jing Zhao* and Songnian He

\author{
"Correspondence: \\ zhaojing200103@163.com \\ College of Science, Civil Aviation \\ University of China, Tianjin, 300300, \\ P.R. China
}

\begin{abstract}
Let $H_{1}, H_{2}, H_{3}$ be real Hilbert spaces, let $A: H_{1} \rightarrow H_{3}, B: H_{2} \rightarrow H_{3}$ be two bounded linear operators. Moudafi introduced simultaneous iterative algorithms (Trans. Math. Program. Appl. 1:1-11, 2013) with weak convergence for the following split common fixed-point problem:

$$
\text { find } x \in F(U), y \in F(T) \text { such that } A x=B y \text {, }
$$

where $U: H_{1} \rightarrow H_{1}$ and $T: H_{2} \rightarrow H_{2}$ are two firmly quasi-nonexpansive operators with nonempty fixed-point sets $F(U)=\left\{x \in H_{1}: U x=x\right\}$ and $F(T)=\left\{x \in H_{2}: T x=x\right\}$. Note that by taking $\mathrm{H}_{2}=\mathrm{H}_{3}$ and $B=1$, we recover the split common fixed-point problem originally introduced in Censor and Segal (J. Convex Anal. 16:587-600, 2009). In this paper, we will continue to consider the split common fixed-point problem (1) governed by the general class of generalized asymptotically quasi-nonexpansive mappings. To estimate the norm of an operator is a very difficult, if it is not an impossible task. The purpose of this paper is to propose a simultaneous iterative algorithm with a way of selecting the stepsizes such that the implementation of the algorithm does not need any prior information as regards the operator norms. MSC: $47 \mathrm{H} 09 ; 47 \mathrm{H} 10 ; 47 \mathrm{J05} ; 54 \mathrm{H} 25$
\end{abstract}

Keywords: split common fixed-point problem; generalized asymptotically quasi-nonexpansive mappings; weak convergence; simultaneous iterative algorithm; Hilbert space

\section{Springer}

\section{Introduction and preliminaries}

Throughout this paper, we always assume that $H$ is a real Hilbert space with the inner product $\langle\cdot, \cdot\rangle$ and the norm $\|\cdot\|$. Let $T: H \rightarrow H$ be a mapping. A point $x \in H$ is said to be a fixed point of $T$ provided $T x=x$. In this paper, we use $F(T)$ to denote the fixed point set and use $\rightarrow$ and $\rightarrow$ to denote the strong convergence and weak convergence, respectively. We use $\omega_{w}\left(x_{k}\right)=\left\{x: \exists x_{k_{j}} \rightarrow x\right\}$ stand for the weak $\omega$-limit set of $\left\{x_{k}\right\}$. 
Let $C$ and $Q$ be nonempty closed convex subset of real Hilbert spaces $H_{1}$ and $H_{2}$, respectively. The split feasibility problem (SFP) is to find a point

$x \in C$ such that $A x \in Q$,

where $A: H_{1} \rightarrow H_{2}$ is a bounded linear operator. The SFP in finite-dimensional Hilbert spaces was first introduced by Censor and Elfving [1] for modeling inverse problems which arise from phase retrievals and in medical image reconstruction [2]. Recently, it has been found that the SFP can also be used in various disciplines such as image restoration, computer tomograph and radiation therapy treatment planning [3-5]. Various algorithms have been invented to solve it, etc. (see [6-9] and references therein).

Note that if the split feasibility problem (1.1) is consistent (i.e., (1.1) has a solution), then (1.1) can be formulated as a fixed point equation by using the fact

$$
P_{C}\left(I-\gamma A^{*}\left(I-P_{Q}\right) A\right) x^{*}=x^{*} .
$$

That is, $x^{*}$ solves the SFP (1.1) if and only if $x^{*}$ solves the fixed point equation (1.2) (see [10] for the details). This implies that we can use fixed point algorithms (see [10-13]) to solve SFP. A popular algorithm that solves the SFP (1.1) is due to Byrne's CQ algorithm [2], which is found to be a gradient-projection method (GPM) in convex minimization.

In [14], Censor and Segal consider the following split common fixed-point problem (SCFP):

$$
\text { find } x^{*} \in F(U) \text { such that } A x^{*} \in F(T) \text {, }
$$

where $A: H_{1} \rightarrow H_{2}$ is a bounded linear operator, $U: H_{1} \rightarrow H_{1}$ and $T: H_{2} \rightarrow H_{2}$ are two nonexpansive operators with nonempty fixed-point sets. To solve (1.3), Censor and Segal [14] proposed and proved, in finite-dimensional spaces, the convergence of the following algorithm:

$$
x_{k+1}=U\left(x_{k}+\gamma A^{t}(T-I) A x_{k}\right), \quad k \in N,
$$

where $\gamma \in\left(0, \frac{2}{\lambda}\right)$, with $\lambda$ being the largest eigenvalue of the matrix $A^{t} A$ ( $A^{t}$ stands for matrix transposition). SCFP (1.3) is in itself at the core of the modeling of many inverse problems in various areas of mathematics and physical sciences and has been used to model significant real-world inverse problems in sensor networks, in radiation therapy treatment planning, in resolution enhancement, in wavelet-based denoising, in antenna design, in computerized tomography, in materials science, in watermarking, in data compression, in magnetic resonance imaging, in holography, in color imaging, in optics and neural networks, in graph matching, etc. (see $[15,16])$.

Let $H_{1}, H_{2}, H_{3}$ be real Hilbert spaces, let $A: H_{1} \rightarrow H_{3}, B: H_{2} \rightarrow H_{3}$ be two bounded linear operators, let $U: H_{1} \rightarrow H_{1}$ and $T: H_{2} \rightarrow H_{2}$ be two firmly quasi-nonexpansive operators. In [17], Moudafi introduced the following split common fixed-point problem:

$$
\text { find } x \in F(U), y \in F(T) \quad \text { such that } \quad A x=B y \text {, }
$$


which allows asymmetric and partial relations between the variables $x$ and $y$. The interest is to cover many situations, for instance, in decomposition methods for PDEs, applications in game theory and in intensity-modulated radiation therapy (IMRT). In decision sciences, this allows to consider agents who interplay only via some components of their decision variables (see [18]). In IMRT, this amounts to envisaging a weak coupling between the vector of doses absorbed in all voxels and that of the radiation intensity (see [3]). If $\mathrm{H}_{2}=\mathrm{H}_{3}$ and $B=I$, then SCFP (1.4) reduces to SCFP (1.3).

For solving the SCFP (1.4), Moudafi [17] introduced the following alternating algorithm:

$$
\left\{\begin{array}{l}
x_{k+1}=U\left(x_{k}-\gamma_{k} A^{*}\left(A x_{k}-B y_{k}\right)\right), \\
y_{k+1}=T\left(y_{k}+\gamma_{k} B^{*}\left(A x_{k+1}-B y_{k}\right)\right)
\end{array}\right.
$$

for firmly quasi-nonexpansive operators $U$ and $T$, where non-decreasing sequence $\gamma_{k} \in$ $\left(\varepsilon, \min \left(\frac{1}{\lambda_{A}}, \frac{1}{\lambda_{B}}\right)-\varepsilon\right), \lambda_{A}, \lambda_{B}$ stand for the spectral radiuses of $A^{*} A$ and $B^{*} B$, respectively.

Very recently, Moudafi [19] introduced the following simultaneous iterative method to solve SCFP (1.4):

$$
\left\{\begin{array}{l}
x_{k+1}=U\left(x_{k}-\gamma_{k} A^{*}\left(A x_{k}-B y_{k}\right)\right), \\
y_{k+1}=T\left(y_{k}+\gamma_{k} B^{*}\left(A x_{k}-B y_{k}\right)\right)
\end{array}\right.
$$

for firmly quasi-nonexpansive operators $U$ and $T$, where $\gamma_{k} \in\left(\varepsilon, \frac{2}{\lambda_{A}+\lambda_{B}}-\varepsilon\right), \lambda_{A}, \lambda_{B}$ stand for the spectral radiuses of $A^{*} A$ and $B^{*} B$, respectively.

Note that in the algorithms (1.5) and (1.6) mentioned above, the determination of the stepsize $\left\{\gamma_{k}\right\}$ depends on the operator (matrix) norms $\|A\|$ and $\|B\|$ (or the largest eigenvalues of $A^{*} A$ and $B^{*} B$ ). In order to implement the alternating algorithm (1.5) and the simultaneous algorithm (1.6) for solving SCFP (1.4), one has first to compute (or, at least, estimate) operator norms of $A$ and $B$, which is in general not an easy work in practice. To overcome this difficulty, López et al. [20] and Zhao and Yang [21] presented a helpful method for estimating the stepsizes which do not need prior knowledge of the operator norms for solving the split feasibility problems and multiple-set split feasibility problems, respectively. Inspired by them, in this paper, we introduce a new choice of the stepsize sequence $\left\{\gamma_{k}\right\}$ for the simultaneous iterative algorithm to solve SCFP (1.4) governed by generalized asymptotically quasi-nonexpansive operators as follows:

$$
\gamma_{k} \in\left(0, \frac{2\left\|A x_{k}-B y_{k}\right\|^{2}}{\left\|A^{*}\left(A x_{k}-B y_{k}\right)\right\|^{2}+\left\|B^{*}\left(A x_{k}-B y_{k}\right)\right\|^{2}}\right) .
$$

The advantage of our choice (1.7) of the stepsizes lies in the fact that no prior information as regards the operator norms of $A$ and $B$ is required, and still convergence is guaranteed.

Now let us recall some definitions, notations and conclusions which will be needed in proving our main results.

Definition 1.1 A mapping $T: H \rightarrow H$ is called demiclosed at the origin if, for any sequence $\left\{x_{n}\right\}$ which weakly converges to $x$, if the sequence $\left\{T x_{n}\right\}$ strongly converges to 0 , then $T x=0$. 
Definition 1.2 (1) A mapping $T: H \rightarrow H$ is said to be nonexpansive if

$$
\|T x-T y\| \leq\|x-y\|, \quad \forall(x, y) \in H \times H .
$$

(2) A mapping $T: H \rightarrow H$ is said to be firmly nonexpansive if

$$
\|T x-T y\|^{2} \leq\|x-y\|^{2}-\|(x-y)-(T x-T y)\|^{2}, \quad \forall(x, y) \in H \times H .
$$

(3) A mapping $T: H \rightarrow H$ is said to be quasi-nonexpansive if $F(T) \neq \emptyset$ such that

$$
\|T x-q\| \leq\|x-q\|, \quad \forall(x, q) \in H \times F(T) .
$$

(4) A mapping $T: H \rightarrow H$ is said to be firmly quasi-nonexpansive if $F(T) \neq \emptyset$ such that

$$
\|T x-q\|^{2} \leq\|x-q\|^{2}-\|x-T x\|^{2}, \quad \forall(x, q) \in H \times F(T) .
$$

(5) A mapping $T: H \rightarrow H$ is said to be asymptotically nonexpansive if there exists a nonnegative real sequence $\left\{l_{k}\right\}$ with $l_{k} \rightarrow 0$ such that for each $k \geq 1$,

$$
\left\|T^{k} x-T^{k} y\right\|^{2} \leq\|x-y\|^{2}+l_{k}\|x-y\|^{2}, \quad \forall(x, y) \in H \times H .
$$

(6) A mapping $T: H \rightarrow H$ is said to be asymptotically quasi-nonexpansive if $F(T) \neq \varnothing$ and there exists a nonnegative real sequence $\left\{l_{k}\right\}$ with $l_{k} \rightarrow 0$ such that for each $k \geq 1$,

$$
\left\|T^{k} x-q\right\|^{2} \leq\|x-q\|^{2}+l_{k}\|x-q\|^{2}, \quad \forall(x, q) \in H \times F(T) .
$$

(7) A mapping $T: H \rightarrow H$ is said to be generalized asymptotically quasi-nonexpansive mapping with $\left(\left\{l_{k}\right\},\left\{\mu_{k}\right\}\right)$ if $F(T) \neq \emptyset$, and there exist nonnegative real sequences $\left\{l_{k}\right\},\left\{\mu_{k}\right\}$ with $l_{k} \rightarrow 0$ and $\mu_{k} \rightarrow 0$ such that for each $k \geq 1$,

$$
\left\|T^{k} x-q\right\|^{2} \leq\|x-q\|^{2}+l_{k}\|x-q\|^{2}+\mu_{k}, \quad \forall(x, q) \in H \times F(T) .
$$

It is clear from this definition that every firmly nonexpansive mapping is nonexpansive, nonexpansive mapping with a fixed point is quasi-nonexpansive, each firmly quasinonexpansive mapping is quasi-nonexpansive, and each quasi-nonexpansive mapping is asymptotically quasi-nonexpansive. We remark that an asymptotically nonexpansive mapping with a nonempty fixed point set $F(T)$ is an asymptotically quasi-nonexpansive mapping, but the converse may be not true. The class of generalized asymptotically quasi-nonexpansive mappings is more general than the class of asymptotically quasinonexpansive mappings and asymptotically nonexpansive mappings. The following example shows that the inclusion is proper. Let $K=\left[-\frac{1}{\pi}, \frac{1}{\pi}\right]$ and define (see [22]) $T x=\frac{x}{2} \sin \left(\frac{1}{x}\right)$ if $x \neq 0$ and $T x=0$ if $x=0$. Then $T^{k} x \rightarrow 0$ uniformly but $T$ is not Lipschitzian. Notice that $F(T)=\{0\}$. For each fixed $k$, define $f_{k}(x)=\left\|T^{k} x\right\|-\|x\|$ for $x \in K$. Set $\mu_{k}=\sup _{x \in K}\left\{f_{k}(x), 0\right\}$. Then $\lim _{k \rightarrow \infty} \mu_{k}=0$ and

$$
\left\|T^{k} x\right\| \leq\|x\|+\mu_{k}
$$


This shows that $T$ is a generalized asymptotically quasi-nonexpansive but it is not asymptotically quasi-nonexpansive and asymptotically nonexpansive because it is not Lipschitzian.

Definition 1.3 A mapping $T: H \rightarrow H$ is said to be uniformly $L$-Lipschitzian if there exists a constant $L>0$ such that for each $k \geq 1$,

$$
\left\|T^{k} x-T^{k} y\right\| \leq L\|x-y\|, \quad \forall(x, y) \in H \times H .
$$

In real Hilbert space, we easily get the following equality:

$$
2\langle x, y\rangle=\|x\|^{2}+\|y\|^{2}-\|x-y\|^{2}=\|x+y\|^{2}-\|x\|^{2}-\|y\|^{2}, \quad \forall x, y \in H .
$$

In what follows, we give some preliminary results needed for the convergence analysis of our algorithms.

Lemma 1.4 ([23]) Let $H$ be a real Hilbert space. Then for all $t \in[0,1]$ and $x, y \in H$,

$$
\|t x+(1-t) y\|^{2}=t\|x\|^{2}+(1-t)\|y\|^{2}-t(1-t)\|x-y\|^{2} .
$$

Lemma 1.5 ([24]) Let $\left\{a_{k}\right\},\left\{b_{k}\right\}$ and $\left\{\delta_{k}\right\}$ be sequences of nonnegative real numbers satisfying

$$
a_{k+1} \leq\left(1+\delta_{k}\right) a_{k}+b_{k}, \quad \forall k \geq 1
$$

If $\sum_{k=1}^{\infty} \delta_{k}<\infty$ and $\sum_{k=1}^{\infty} b_{k}<\infty$, then the limit $\lim _{k \rightarrow \infty} a_{k}$ exists.

\section{Simultaneous iterative algorithm without prior knowledge of operator norms}

In this section we introduce a simultaneous iterative algorithm where the stepsizes don't depend on the operator norms $\|A\|$ and $\|B\|$ and prove weak convergence of the algorithm to solve SCFP (1.4) governed by generalized asymptotically quasi-nonexpansive operators.

We always assume that $H_{1}, H_{2}, H_{3}$ are real Hilbert spaces and $A: H_{1} \rightarrow H_{3}, B: H_{2} \rightarrow H_{3}$ are two bounded linear operators. Let $U: H_{1} \rightarrow H_{1}$ and $T: H_{2} \rightarrow H_{2}$ be two generalized asymptotically quasi-nonexpansive mappings with $\left(\left\{l_{k}\right\},\left\{\mu_{k}\right\}\right)$. In the sequel, we use $\Gamma$ to denote the set of solutions of SCFP (1.4), i.e.,

$$
\Gamma=\{x \in F(U), y \in F(T) \text { such that } A x=B y\} .
$$

Algorithm 2.1 Let $x_{0} \in H_{1}, y_{0} \in H_{2}$ be arbitrary and $\alpha_{k} \in[0,1]$. Assume that the $k$ th iterate $x_{k} \in H_{1}, y_{k} \in H_{2}$ has been constructed; then we calculate the $(k+1)$ th iterate $\left(x_{k+1}, y_{k+1}\right)$ via the formula:

$$
\left\{\begin{array}{l}
u_{k}=x_{k}-\gamma_{k} A^{*}\left(A x_{k}-B y_{k}\right), \\
x_{k+1}=\alpha_{k} u_{k}+\left(1-\alpha_{k}\right) U^{k}\left(u_{k}\right), \\
v_{k}=y_{k}+\gamma_{k} B^{*}\left(A x_{k}-B y_{k}\right), \\
y_{k+1}=\alpha_{k} v_{k}+\left(1-\alpha_{k}\right) T^{k}\left(v_{k}\right) .
\end{array}\right.
$$


The stepsize $\gamma_{k}$ is chosen in such a way that

$$
\gamma_{k} \in\left(0, \frac{2\left\|A x_{k}-B y_{k}\right\|^{2}}{\left\|A^{*}\left(A x_{k}-B y_{k}\right)\right\|^{2}+\left\|B^{*}\left(A x_{k}-B y_{k}\right)\right\|^{2}}\right), \quad k \in \Omega,
$$

otherwise, $\gamma_{k}=\gamma$ ( $\gamma$ being any nonnegative value), where the set of indices $\Omega=\left\{k: A x_{k}-\right.$ $\left.B y_{k} \neq 0\right\}$.

Remark 2.2 Note that in (2.2) the choice of the stepsize $\gamma_{k}$ is independent of the norms $\|A\|$ and $\|B\|$. The value of $\gamma$ does not influence the considered algorithm, but it was introduced just for the sake of clarity. Furthermore, we will see from Lemma 2.3 that $\gamma_{k}$ is well defined.

Lemma 2.3 If $\Gamma$ is nonempty, then $\gamma_{k}$ defined by (2.2) is well defined.

Proof Taking $(x, y) \in \Gamma$, i.e., $x \in F(U), y \in F(T)$ and $A x=B y$. We have

$$
\left\langle A^{*}\left(A x_{k}-B y_{k}\right), x_{k}-x\right\rangle=\left\langle A x_{k}-B y_{k}, A x_{k}-A x\right\rangle
$$

and

$$
\left\langle B^{*}\left(A x_{k}-B y_{k}\right), y-y_{k}\right\rangle=\left\langle A x_{k}-B y_{k}, B y-B y_{k}\right\rangle
$$

By adding the two above equalities and by taking into account the fact that $A x=B y$, we obtain

$$
\begin{aligned}
\left\|A x_{k}-B y_{k}\right\|^{2} & =\left\langle A^{*}\left(A x_{k}-B y_{k}\right), x_{k}-x\right\rangle+\left\langle B^{*}\left(A x_{k}-B y_{k}\right), y-y_{k}\right\rangle \\
& \leq\left\|A^{*}\left(A x_{k}-B y_{k}\right)\right\| \cdot\left\|x_{k}-x\right\|+\left\|B^{*}\left(A x_{k}-B y_{k}\right)\right\| \cdot\left\|y-y_{k}\right\| .
\end{aligned}
$$

Consequently, for $k \in \Omega$, that is, $\left\|A x_{k}-B y_{k}\right\|>0$, we have $\left\|A^{*}\left(A x_{k}-B y_{k}\right)\right\| \neq 0$ or $\| B^{*}\left(A x_{k}-\right.$ $\left.B y_{k}\right) \| \neq 0$. This leads to the conclusion that $\gamma_{k}$ is well defined.

Theorem 2.4 Assume that $U-I, T-I$ are demiclosed at origin, and $U, T$ are uniformly L-Lipschitzian. Let the sequence $\left\{\left(x_{k}, y_{k}\right)\right\}$ be generated by Algorithm 2.1. Assume $\Gamma$ is nonempty and for small enough $\epsilon>0$,

$$
\gamma_{k} \in\left(\epsilon, \frac{2\left\|A x_{k}-B y_{k}\right\|^{2}}{\left\|A^{*}\left(A x_{k}-B y_{k}\right)\right\|^{2}+\left\|B^{*}\left(A x_{k}-B y_{k}\right)\right\|^{2}}-\epsilon\right)
$$

where $k \in \Omega$. Then $\left\{\left(x_{k}, y_{k}\right)\right\}$ weakly converges to a solution $\left(x^{*}, y^{*}\right)$ of $(1.4)$ provided that $\sum_{k=1}^{\infty}\left(l_{k}+\mu_{k}\right)<\infty$ and $\left\{\alpha_{k}\right\} \subset(\delta, 1-\delta)$ for small enough $\delta>0$. Moreover, $\left\{x_{k}\right\}$ and $\left\{y_{k}\right\}$ are asymptotically regular and $\left\|A x_{k}-B y_{k}\right\| \rightarrow 0$.

Proof From the condition on $\gamma_{k}$, we have

$$
\inf _{k \in \Omega}\left\{\frac{2\left\|A x_{k}-B y_{k}\right\|^{2}}{\left\|A^{*}\left(A x_{k}-B y_{k}\right)\right\|^{2}+\left\|B^{*}\left(A x_{k}-B y_{k}\right)\right\|^{2}}-\gamma_{k}\right\}>0
$$


On the other hand, from $\left\|A^{*}\left(A x_{k}-B y_{k}\right)\right\|^{2} \leq\left\|A^{*}\right\|^{2}\left\|A x_{k}-B y_{k}\right\|^{2}$ and $\left\|B^{*}\left(A x_{k}-B y_{k}\right)\right\|^{2} \leq$ $\left\|B^{*}\right\|^{2}\left\|A x_{k}-B y_{k}\right\|^{2}$ we obtain $\left\{\frac{2\left\|A x_{k}-B y_{k}\right\|^{2}}{\left\|A^{*}\left(A x_{k}-B y_{k}\right)\right\|^{2}+\left\|B^{*}\left(A x_{k}-B y_{k}\right)\right\|^{2}}\right\}$ is lower bounded by $\frac{2}{\|A\|^{2}+\|B\|^{2}}$ and so

$$
\inf _{k \in \Omega}\left\{\frac{2\left\|A x_{k}-B y_{k}\right\|^{2}}{\left\|A^{*}\left(A x_{k}-B y_{k}\right)\right\|^{2}+\left\|B^{*}\left(A x_{k}-B y_{k}\right)\right\|^{2}}\right\} \geq \frac{2}{\|A\|^{2}+\|B\|^{2}}>-\infty .
$$

It follows from (2.3) that $\sup _{k \in \Omega} \gamma_{k}<+\infty$ and $\left\{\gamma_{k}\right\}_{k \geq 1}$ is bounded.

Taking $(x, y) \in \Gamma$, i.e., $x \in F(U) ; y \in F(T)$ and $A x=B y$. We have

$$
\begin{aligned}
\left\|u_{k}-x\right\|^{2} & =\left\|x_{k}-\gamma_{k} A^{*}\left(A x_{k}-B y_{k}\right)-x\right\|^{2} \\
& =\left\|x_{k}-x\right\|^{2}-2 \gamma_{k}\left(x_{k}-x, A^{*}\left(A x_{k}-B y_{k}\right)\right\rangle+\gamma_{k}^{2}\left\|A^{*}\left(A x_{k}-B y_{k}\right)\right\|^{2} .
\end{aligned}
$$

Using equality (1.8), we have

$$
\begin{aligned}
-2\left\langle x_{k}-x, A^{*}\left(A x_{k}-B y_{k}\right)\right\rangle & =-2\left\langle A x_{k}-A x, A x_{k}-B y_{k}\right\rangle \\
& =-\left\|A x_{k}-A x\right\|^{2}-\left\|A x_{k}-B y_{k}\right\|^{2}+\left\|B y_{k}-A x\right\|^{2} .
\end{aligned}
$$

By (2.4) and (2.5), we obtain

$$
\begin{aligned}
\left\|u_{k}-x\right\|^{2}= & \left\|x_{k}-x\right\|^{2}-\gamma_{k}\left\|A x_{k}-A x\right\|^{2}-\gamma_{k}\left\|A x_{k}-B y_{k}\right\|^{2} \\
& +\gamma_{k}\left\|B y_{k}-A x\right\|^{2}+\gamma_{k}^{2}\left\|A^{*}\left(A x_{k}-B y_{k}\right)\right\|^{2} .
\end{aligned}
$$

Similarly, by (2.1) we have

$$
\begin{aligned}
\left\|v_{k}-y\right\|^{2}= & \left\|y_{k}-y\right\|^{2}-\gamma_{k}\left\|B y_{k}-B y\right\|^{2}-\gamma_{k}\left\|A x_{k}-B y_{k}\right\|^{2} \\
& +\gamma_{k}\left\|A x_{k}-B y\right\|^{2}+\gamma_{k}^{2}\left\|B^{*}\left(A x_{k}-B y_{k}\right)\right\|^{2} .
\end{aligned}
$$

By adding the two last inequalities, and by taking into account fact that $A x=B y$, we obtain

$$
\begin{aligned}
&\left\|u_{k}-x\right\|^{2}+\left\|v_{k}-y\right\|^{2} \\
&=\left\|x_{k}-x\right\|^{2}+\left\|y_{k}-y\right\|^{2} \\
& \quad-\gamma_{k}\left[2\left\|A x_{k}-B y_{k}\right\|^{2}-\gamma_{k}\left(\left\|A^{*}\left(A x_{k}-B y_{k}\right)\right\|^{2}+\left\|B^{*}\left(A x_{k}-B y_{k}\right)\right\|^{2}\right)\right] .
\end{aligned}
$$

By the fact that $U$ and $T$ are generalized asymptotically quasi-nonexpansive mappings with $\left(\left\{l_{k}\right\},\left\{\mu_{k}\right\}\right)$, it follows from Lemma 1.4 that

$$
\begin{aligned}
\left\|x_{k+1}-x\right\|^{2}= & \alpha_{k}\left\|u_{k}-x\right\|^{2}+\left(1-\alpha_{k}\right)\left\|U^{k}\left(u_{k}\right)-x\right\|^{2}-\alpha_{k}\left(1-\alpha_{k}\right)\left\|U^{k}\left(u_{k}\right)-u_{k}\right\|^{2} \\
\leq & \alpha_{k}\left\|u_{k}-x\right\|^{2}+\left(1-\alpha_{k}\right)\left\|u_{k}-x\right\|^{2}+\left(1-\alpha_{k}\right) l_{k}\left\|u_{k}-x\right\|^{2} \\
& +\left(1-\alpha_{k}\right) \mu_{k}-\alpha_{k}\left(1-\alpha_{k}\right)\left\|U^{k}\left(u_{k}\right)-u_{k}\right\|^{2} \\
= & \left\|u_{k}-x\right\|^{2}+\left(1-\alpha_{k}\right) l_{k}\left\|u_{k}-x\right\|^{2}+\left(1-\alpha_{k}\right) \mu_{k}-\alpha_{k}\left(1-\alpha_{k}\right)\left\|U^{k}\left(u_{k}\right)-u_{k}\right\|^{2}
\end{aligned}
$$


and

$$
\left\|y_{k+1}-y\right\|^{2} \leq\left\|v_{k}-y\right\|^{2}+\left(1-\alpha_{k}\right) l_{k}\left\|v_{k}-y\right\|^{2}+\left(1-\alpha_{k}\right) \mu_{k}-\alpha_{k}\left(1-\alpha_{k}\right)\left\|T^{k}\left(v_{k}\right)-v_{k}\right\|^{2} .
$$

So, by (2.8), we have

$$
\begin{aligned}
& \left\|x_{k+1}-x\right\|^{2}+\left\|y_{k+1}-y\right\|^{2} \\
& \leq\left[1+\left(1-\alpha_{k}\right) l_{k}\right]\left(\left\|x_{k}-x\right\|^{2}+\left\|y_{k}-y\right\|^{2}\right)+2\left(1-\alpha_{k}\right) \mu_{k} \\
& \quad-\gamma_{k}\left[1+\left(1-\alpha_{k}\right) l_{k}\right]\left[2\left\|A x_{k}-B y_{k}\right\|^{2}-\gamma_{k}\left(\left\|A^{*}\left(A x_{k}-B y_{k}\right)\right\|^{2}+\left\|B^{*}\left(A x_{k}-B y_{k}\right)\right\|^{2}\right)\right] \\
& \quad-\alpha_{k}\left(1-\alpha_{k}\right)\left\|U^{k}\left(u_{k}\right)-u_{k}\right\|^{2}-\alpha_{k}\left(1-\alpha_{k}\right)\left\|T^{k}\left(v_{k}\right)-v_{k}\right\|^{2} .
\end{aligned}
$$

Now, by setting $\rho_{k}(x, y):=\left\|x_{k}-x\right\|^{2}+\left\|y_{k}-y\right\|^{2}$, we obtain the following inequality:

$$
\begin{aligned}
& \rho_{k+1}(x, y) \\
& \leq\left(1+\xi_{k}\right) \rho_{k}(x, y)+\eta_{k} \\
& \quad-\gamma_{k}\left(1+\xi_{k}\right)\left[2\left\|A x_{k}-B y_{k}\right\|^{2}-\gamma_{k}\left(\left\|A^{*}\left(A x_{k}-B y_{k}\right)\right\|^{2}+\left\|B^{*}\left(A x_{k}-B y_{k}\right)\right\|^{2}\right)\right] \\
& \quad-\alpha_{k}\left(1-\alpha_{k}\right)\left\|U^{k}\left(u_{k}\right)-u_{k}\right\|^{2}-\alpha_{k}\left(1-\alpha_{k}\right)\left\|T^{k}\left(v_{k}\right)-v_{k}\right\|^{2},
\end{aligned}
$$

where $\xi_{k}=\left(1-\alpha_{k}\right) l_{k}$ and $\eta_{k}=2\left(1-\alpha_{k}\right) \mu_{k}$. By the condition $\sum_{k=1}^{\infty}\left(l_{k}+\mu_{k}\right)<\infty$, we have $\sum_{k=1}^{\infty} \xi_{k}<\infty$ and $\sum_{k=1}^{\infty} \eta_{k}<\infty$. It follows from the condition on $\left\{\gamma_{k}\right\}$ that

$$
\rho_{k+1}(x, y) \leq\left(1+\xi_{k}\right) \rho_{k}(x, y)+\eta_{k}
$$

By Lemma 1.5, the following limit exists:

$$
\lim _{k \rightarrow \infty} \rho_{k}(x, y):=\rho(x, y)
$$

So the sequences $\left\{x_{k}\right\}$ and $\left\{y_{k}\right\}$ are bounded. Now, we rewrite (2.10) as follows:

$$
\begin{aligned}
\gamma_{k}(1+ & \left.\xi_{k}\right)\left[2\left\|A x_{k}-B y_{k}\right\|^{2}-\gamma_{k}\left(\left\|A^{*}\left(A x_{k}-B y_{k}\right)\right\|^{2}+\left\|B^{*}\left(A x_{k}-B y_{k}\right)\right\|^{2}\right)\right] \\
& +\alpha_{k}\left(1-\alpha_{k}\right)\left\|U^{k}\left(u_{k}\right)-u_{k}\right\|^{2}+\alpha_{k}\left(1-\alpha_{k}\right)\left\|T^{k}\left(v_{k}\right)-v_{k}\right\|^{2} \\
\leq & \rho_{k}(x, y)-\rho_{k+1}(x, y)+\xi_{k} \rho_{k}(x, y)+\eta_{k} .
\end{aligned}
$$

It follows from the assumption

$$
\gamma_{k} \in\left(\epsilon, \frac{2\left\|A x_{k}-B y_{k}\right\|^{2}}{\left\|A^{*}\left(A x_{k}-B y_{k}\right)\right\|^{2}+\left\|B^{*}\left(A x_{k}-B y_{k}\right)\right\|^{2}}-\epsilon\right), \quad k \in \Omega
$$

that

$$
\lim _{k \rightarrow \infty}\left(\left\|A^{*}\left(A x_{k}-B y_{k}\right)\right\|^{2}+\left\|B^{*}\left(A x_{k}-B y_{k}\right)\right\|^{2}\right)=0 .
$$


(Note that $A x_{k}-B y_{k}=0$ if $k \notin \Omega$.) So, we obtain

$$
\lim _{k \rightarrow \infty}\left\|A^{*}\left(A x_{k}-B y_{k}\right)\right\|=\lim _{k \rightarrow \infty}\left\|B^{*}\left(A x_{k}-B y_{k}\right)\right\|=0
$$

Similarly, by the conditions on $\left\{\alpha_{k}\right\}$, we obtain

$$
\lim _{k \rightarrow \infty}\left\|U^{k}\left(u_{k}\right)-u_{k}\right\|=\lim _{k \rightarrow \infty}\left\|T^{k}\left(v_{k}\right)-v_{k}\right\|=0
$$

Using the assumption on $\gamma_{k},(2.11),(2.12)$, and the convergence of $\rho_{k}(x, y)$ we have

$$
\lim _{k \rightarrow \infty}\left\|A x_{k}-B y_{k}\right\|=0
$$

Since

$$
\left\|u_{k}-x_{k}\right\|=\gamma_{k}\left\|A^{*}\left(A x_{k}-B y_{k}\right)\right\|
$$

and $\left\{\gamma_{k}\right\}$ is bounded, we have $\lim _{k \rightarrow \infty}\left\|u_{k}-x_{k}\right\|=0$. It follows from $\lim _{k \rightarrow \infty}\left\|U^{k}\left(u_{k}\right)-u_{k}\right\|=$ 0 that $\lim _{k \rightarrow \infty}\left\|U^{k}\left(u_{k}\right)-x_{k}\right\|=0$. So,

$$
\left\|x_{k+1}-x_{k}\right\| \leq \alpha_{k}\left\|u_{k}-x_{k}\right\|+\left(1-\alpha_{k}\right)\left\|U^{k}\left(u_{k}\right)-x_{k}\right\| \rightarrow 0
$$

as $k \rightarrow \infty$, from which one infers that $\left\{x_{k}\right\}$ is asymptotically regular, namely $\lim _{k \rightarrow \infty} \| x_{k+1}-$ $x_{k} \|=0$. Noting

$$
\begin{aligned}
\left\|u_{k+1}-u_{k}\right\| & =\left\|x_{k+1}-x_{k}-\gamma_{k+1} A^{*}\left(A x_{k+1}-B y_{k+1}\right)+\gamma_{k} A^{*}\left(A x_{k}-B y_{k}\right)\right\| \\
& \leq\left\|x_{k+1}-x_{k}\right\|+\gamma_{k+1}\left\|A^{*}\left(A x_{k+1}-B y_{k+1}\right)\right\|+\gamma_{k}\left\|A^{*}\left(A x_{k}-B y_{k}\right)\right\|,
\end{aligned}
$$

from (2.14) and (2.15) we have

$$
\lim _{k \rightarrow \infty}\left\|u_{k+1}-u_{k}\right\|=0
$$

Similarly, $\lim _{k \rightarrow \infty}\left\|v_{k}-y_{k}\right\|=0,\left\{y_{k}\right\}$ and $\left\{v_{k}\right\}$ are asymptotically regular, too.

Next, we prove that $\left\|u_{k}-U\left(u_{k}\right)\right\| \rightarrow 0$ and $\left\|v_{k}-T\left(v_{k}\right)\right\| \rightarrow 0$ as $k \rightarrow \infty$. In fact, since $U$ is uniformly $L$-Lipschitzian continuous, it follows from (2.13) and (2.16) that

$$
\begin{aligned}
& \left\|u_{k}-U\left(u_{k}\right)\right\| \\
& \quad \leq\left\|u_{k}-U^{k}\left(u_{k}\right)\right\|+\left\|U^{k}\left(u_{k}\right)-U\left(u_{k}\right)\right\| \\
& \quad \leq\left\|u_{k}-U^{k}\left(u_{k}\right)\right\|+L\left\|U^{k-1}\left(u_{k}\right)-u_{k}\right\| \\
& \quad \leq\left\|u_{k}-U^{k}\left(u_{k}\right)\right\|+L\left(\left\|U^{k-1}\left(u_{k}\right)-U^{k-1}\left(u_{k-1}\right)\right\|+\left\|U^{k-1}\left(u_{k-1}\right)-u_{k}\right\|\right) \\
& \quad \leq\left\|u_{k}-U^{k}\left(u_{k}\right)\right\|+L\left(L\left\|u_{k}-u_{k-1}\right\|+\left\|U^{k-1}\left(u_{k-1}\right)-u_{k-1}\right\|+\left\|u_{k-1}-u_{k}\right\|\right) \rightarrow 0 .
\end{aligned}
$$

Since $T$ is uniformly $L$-Lipschitzian continuous, in the same way as above, we can also prove that $\left\|v_{k}-T\left(v_{k}\right)\right\| \rightarrow 0$ as $k \rightarrow \infty$. 
Taking $\left(x^{*}, y^{*}\right) \in \omega_{w}\left(x_{k}, y_{k}\right)$, from $\lim _{k \rightarrow \infty}\left\|u_{k}-x_{k}\right\|=0$ and $\lim _{k \rightarrow \infty}\left\|v_{k}-y_{k}\right\|=0$, we have $x^{*} \in \omega_{w}\left(u_{k}\right)$ and $y^{*} \in \omega_{w}\left(v_{k}\right)$. Combined with the demiclosednesses of $U-I$ and $T-I$ at 0 ,

$$
\lim _{k \rightarrow \infty}\left\|U\left(u_{k}\right)-u_{k}\right\|=\lim _{k \rightarrow \infty}\left\|T\left(v_{k}\right)-v_{k}\right\|=0
$$

yields $U x^{*}=x^{*}$ and $T y^{*}=y^{*}$. So, $x^{*} \in F(U)$ and $y^{*} \in F(T)$. On the other hand, $A x^{*}-B y^{*} \in$ $\omega_{w}\left(A x_{k}-B y_{k}\right)$ and weakly lower semicontinuity of the norm imply that

$$
\left\|A x^{*}-B y^{*}\right\| \leq \liminf _{k \rightarrow \infty}\left\|A x_{k}-B y_{k}\right\|=0,
$$

hence $\left(x^{*}, y^{*}\right) \in \Gamma$.

Finally, we will show the uniqueness of the weak cluster points of $\left\{x_{k}\right\}$ and $\left\{y_{k}\right\}$. Indeed, let $\bar{x}, \bar{y}$ be other weak cluster points of $\left\{x_{k}\right\}$ and $\left\{y_{k}\right\}$, respectively, then $(\bar{x}, \bar{y}) \in \Gamma$. From the definition of $\rho_{k}(x, y)$, we have

$$
\begin{aligned}
& \rho_{k}\left(x^{*}, y^{*}\right) \\
& \quad=\left\|x_{k}-\bar{x}\right\|^{2}+\left\|\bar{x}-x^{*}\right\|^{2}+2\left\langle x_{k}-\bar{x}, \bar{x}-x^{*}\right\rangle+\left\|y_{k}-\bar{y}\right\|^{2}+\left\|\bar{y}-y^{*}\right\|^{2}+2\left\langle y_{k}-\bar{y}, \bar{y}-y^{*}\right\rangle \\
& \quad=\rho_{k}(\bar{x}, \bar{y})+\left\|\bar{x}-x^{*}\right\|^{2}+\left\|\bar{y}-y^{*}\right\|^{2}+2\left\langle x_{k}-\bar{x}, \bar{x}-x^{*}\right\rangle+2\left\langle y_{k}-\bar{y}, \bar{y}-y^{*}\right\rangle .
\end{aligned}
$$

Without loss of generality, we may assume that $x_{k}-\bar{x}$ and $y_{k} \rightarrow \bar{y}$. By passing to the limit in relation (2.17), we obtain

$$
\rho\left(x^{*}, y^{*}\right)=\rho(\bar{x}, \bar{y})+\left\|\bar{x}-x^{*}\right\|^{2}+\left\|\bar{y}-y^{*}\right\|^{2} .
$$

Reversing the role of $\left(x^{*}, y^{*}\right)$ and $(\bar{x}, \bar{y})$, we also have

$$
\rho(\bar{x}, \bar{y})=\rho\left(x^{*}, y^{*}\right)+\left\|x^{*}-\bar{x}\right\|^{2}+\left\|y^{*}-\bar{y}\right\|^{2} .
$$

By adding the two last equalities, we obtain $x^{*}=\bar{x}$ and $y^{*}=\bar{y}$, which implies that the whole sequence $\left\{\left(x_{k}, y_{k}\right)\right\}$ weakly converges to a solution of problem (1.4). This completes the proof.

The following conclusions can be obtained from Theorem 2.4 immediately.

Theorem 2.5 Let $U: H_{1} \rightarrow H_{1}$ and $T: H_{2} \rightarrow H_{2}$ be two asymptotically quasinonexpansive mappings with $\left(\left\{l_{k}\right\}\right)$. Assume that $U-I, T-I$ are demiclosed at origin, and $U, T$ are uniformly L-Lipschitzian. Let the sequence $\left\{\left(x_{k}, y_{k}\right)\right\}$ be generated by Algorithm 2.1. Assume $\Gamma$ is nonempty and for small enough $\epsilon>0$,

$$
\gamma_{k} \in\left(\epsilon, \frac{2\left\|A x_{k}-B y_{k}\right\|^{2}}{\left\|A^{*}\left(A x_{k}-B y_{k}\right)\right\|^{2}+\left\|B^{*}\left(A x_{k}-B y_{k}\right)\right\|^{2}}-\epsilon\right)
$$

where $k \in \Omega$. Then $\left\{\left(x_{k}, y_{k}\right)\right\}$ weakly converges to a solution $\left(x^{*}, y^{*}\right)$ of $(1.4)$ provided that $\sum_{k=1}^{\infty} l_{k}<\infty$ and $\left\{\alpha_{k}\right\} \subset(\delta, 1-\delta)$ for small enough $\delta>0$. Moreover, $\left\{x_{k}\right\}$ and $\left\{y_{k}\right\}$ are asymptotically regular and $\left\|A x_{k}-B y_{k}\right\| \rightarrow 0$. 
Theorem 2.6 Let $U: H_{1} \rightarrow H_{1}$ and $T: H_{2} \rightarrow H_{2}$ be two quasi-nonexpansive mappings. Assume that $U-I, T-I$ are demiclosed at origin, and $U, T$ are uniformly L-Lipschitzian. Let the sequence $\left\{\left(x_{k}, y_{k}\right)\right\}$ be generated by Algorithm 2.1. Assume $\Gamma$ is nonempty and for small enough $\epsilon>0$,

$$
\gamma_{k} \in\left(\epsilon, \frac{2\left\|A x_{k}-B y_{k}\right\|^{2}}{\left\|A^{*}\left(A x_{k}-B y_{k}\right)\right\|^{2}+\left\|B^{*}\left(A x_{k}-B y_{k}\right)\right\|^{2}}-\epsilon\right),
$$

where $k \in \Omega$. Then $\left\{\left(x_{k}, y_{k}\right)\right\}$ weakly converges to a solution $\left(x^{*}, y^{*}\right)$ of $(1.4)$ provided that $\left\{\alpha_{k}\right\} \subset(\delta, 1-\delta)$ for small enough $\delta>0$. Moreover, $\left\{x_{k}\right\}$ and $\left\{y_{k}\right\}$ are asymptotically regular and $\left\|A x_{k}-B y_{k}\right\| \rightarrow 0$.

Remark 2.7 When $B=I$, Algorithm 2.1 becomes

$$
\left\{\begin{array}{l}
u_{k}=x_{k}-\gamma_{k} A^{*}\left(A x_{k}-y_{k}\right), \\
x_{k+1}=\alpha_{k} u_{k}+\left(1-\alpha_{k}\right) U^{k}\left(u_{k}\right), \\
v_{k}=\left(1-\gamma_{k}\right) y_{k}+\gamma_{k} A x_{k}, \\
y_{k+1}=\beta_{k} v_{k}+\left(1-\beta_{k}\right) T^{k}\left(v_{k}\right),
\end{array}\right.
$$

where the stepsize $\gamma_{k}$ is chosen in such a way that

$$
\gamma_{k} \in\left(0, \frac{2\left\|A x_{k}-B y_{k}\right\|^{2}}{\left\|A^{*}\left(A x_{k}-B y_{k}\right)\right\|^{2}+\left\|A x_{k}-B y_{k}\right\|^{2}}\right), \quad k \in \Omega,
$$

otherwise $\gamma_{k}=\gamma$ ( $\gamma$ being any nonnegative value), where the set of indices $\Omega=\{k$ : $\left.A x_{k}-y_{k} \neq 0\right\}$. This solves SCFP (1.3) for generalized asymptotically quasi-nonexpansive operators, asymptotically quasi-nonexpansive operators, quasi-nonexpansive operators, and firmly quasi-nonexpansive operators without prior knowledge of operator norm $\|A\|$.

\section{Competing interests}

The authors declare that they have no competing interests.

\section{Authors' contributions}

All authors contributed equally to the writing of this paper. All authors read and approved the final manuscript.

\section{Acknowledgements}

The research was supported by the Fundamental Research Funds for the Central Universities (Program No. 3122013k004), it was also supported by the Fundamental Research Funds for the Central Universities (Program No. 3122013C002).

Received: 21 November 2013 Accepted: 6 March 2014 Published: 25 Mar 2014

\section{References}

1. Censor, Y, Elfving, T: A multiprojection algorithm using Bregman projections in a product space. Numer. Algorithms 8 , 221-239 (1994)

2. Byrne, C: Iterative oblique projection onto convex subsets and the split feasibility problem. Inverse Probl. 18, 441-453 (2002)

3. Censor, Y, Bortfeld, T, Martin, B, Trofimov, A: A unified approach for inversion problems in intensity-modulated radiation therapy. Phys. Med. Biol. 51, 2353-2365 (2006)

4. Censor, Y, Elfving, T, Kopf, N, Bortfeld, T: The multiple-sets split feasibility problem and its applications. Inverse Probl. 21, 2071-2084 (2005)

5. Censor, Y, Motova, A, Segal, A: Perturbed projections and subgradient projections for the multiple-setssplit feasibility problem. J. Math. Anal. Appl. 327, 1244-1256 (2007)

6. Byrne, C: A unified treatment of some iterative algorithms in signal processing and image reconstruction. Inverse Probl. 20, 103-120 (2004) 
7. Qu, B, Xiu, N: A note on the CQ algorithm for the split feasibility problem. Inverse Probl. 21, 1655-1665 (2005)

8. Yao, Y, Wu, J, Liou, YC: Regularized methods for the split feasibility problem. Abstr. Appl. Anal. 2012, Article ID 140679 (2012)

9. Yao, Y, Postolache, M, Liou, YC: Strong convergence of a self-adaptive method for the split feasibility problem. Fixed Point Theory Appl. 2013, ID 201 (2013)

10. Xu, HK: Iterative methods for the split feasibility problem in infinite-dimensional Hilbert spaces. Inverse Probl. 26(10), 105018 (2010)

11. Bauschke, HH, Borwein, JM: On projection algorithms for solving convex feasibility problems. SIAM Rev. 38(3), 367-426 (1996)

12. Xu, HK: A variable Krasnosel'skii-Mann algorithm and the multiple-set split feasibility problem. Inverse Probl. 22, 2021-2034 (2006)

13. Masad, E, Reich, S: A note on the multiple-set split convex feasibility problem in Hilbert space. J. Nonlinear Convex Anal. 8, 367-371 (2007)

14. Censor, Y, Segal, A: The split common fixed point problem for directed operators. J. Convex Anal. 16, 587-600 (2009)

15. Censor, Y, Gibali, A, Reich, S: Algorithms for the split variational inequality problem. Numer. Algorithms 59, $301-323$ (2012)

16. Byrne, C, Censor, Y, Gibali, A, Reich, S: The split common null point problem. J. Nonlinear Convex Anal. 13, 759-775 (2012)

17. Moudafi, A: Alternating CQ-algorithm for convex feasibility and split fixed-point problems. J. Nonlinear and Convex Anal. (submitted for publication)

18. Attouch, H, Bolte, J, Redont, P, Soubeyran, A: Alternating proximal algorithms for weakly coupled minimization problems. Applications to dynamical games and PDEs. J. Convex Anal. 15, 485-506 (2008)

19. Moudafi, A, Al-Shemas, E: Simultaneous iterative methods for split equality problems and application. Trans. Math Program. Appl. 1, 1-11 (2013)

20. López, G, Martín-Márquez, V, Wang, F, Xu, HK: Solving the split feasibility problem without prior knowledge of matrix norms. Inverse Probl. 27, 085004 (2012)

21. Zhao, J, Yang, Q: A simple projection method for solving the multiple-sets split feasibility problem. Inverse Probl. Sci. Eng. 21(3), 537-546 (2013)

22. Shahzad, $\mathrm{N}$, Zegeye, $\mathrm{H}$ : Strong convergence of implicit iteration process for a finite family of generalized asymptotically quasi-nonexpansive maps. Appl. Math. Comput. 189, 1058-1065 (2007)

23. Matinez-Yanes, C, Xu, HK: Strong convergence of the CQ method for fixed point processes. Nonlinear Anal. 64, 2400-2411 (2006)

24. Aoyama, K, Kimura, W, Takahashi, W, Toyoda, M: Approximation of common fixed points of acountable family of nonexpansive mappings on a Banach space. Nonlinear Anal. 67(8), 2350-2360 (2007)

10.1186/1687-1812-2014-73

Cite this article as: Zhao and He: Simultaneous iterative algorithms for the split common fixed-point problem of generalized asymptotically quasi-nonexpansive mappings without prior knowledge of operator norms. Fixed Point Theory and Applications 2014, 2014:73

\section{Submit your manuscript to a SpringerOpen ${ }^{\odot}$ journal and benefit from:}

- Convenient online submission

Rigorous peer review

- Immediate publication on acceptance

- Open access: articles freely available online

- High visibility within the field

- Retaining the copyright to your article 